\title{
Organizing pneumonia as the initial presentation in rheumatoid arthritis \\ - A case report
}

\author{
Harveen Kaur ${ }^{1}$, Dilbag Singh ${ }^{1}$ and Naveen Pandhi ${ }^{2}$ \\ ${ }^{1} J u n i o r$ Resident, Department of Pulmonary Medicine, Government Medical College, Amritsar, \\ Punjab, India \\ ${ }^{2}$ Professor and Head, Department of Pulmonary Medicine, Government Medical College, Amritsar, \\ Punjab, India
}

\section{Abstract \\ Organizing pneumonia (OP), can be seen in association with lung injury, infection, drug intoxication, and connective tissue diseases. Patients of rheumatoid arthritis (RA) are prone to develop interstitial lung disease (ILD), but the pulmonary involvement usually occurs several years after the joint manifestations. Only in about $10 \%$ cases of RA, the initial manifestation of the disease can be in the form of interstitial lung disease. OP as the initial manifestation of RA is extremely uncommon occurrence.}

Here is presented a case of 52-year-old male who presented with OP as the initial manifestation of RA. On investigation, the RA factor and anti-CCP Antibodies were positive. Based on clinical, radiological and histopathological findings the diagnosis was established.

\section{More Information}

*Address for Correspondence: Harveen Kaur, Junior Resident, Department of Pulmonary Medicine, Government Medical College, Amritsar, Punjab, India, Email: hk_94basra@ymail.com

Submitted: June 14, 2021

Approved: June 23, 2021

Published: June 25, 2021

How to cite this article: Kaur H, Singh D, Pandhi N. Organizing pneumonia as the initial presentation in rheumatoid arthritis - A case report. J Pulmonol Respir Res. 2021; 5: 051-053.

DOI: 10.29328/journal.jprr.1001024

Copyright: () $2021 \mathrm{Kaur} \mathrm{H}$, et al. This is an open access article distributed under the Creative Commons Attribution License, which permits unrestricted use, distribution, and reproduction in any medium, provided the original work is properly cited.

Keywords: Rheumatoid arthritis (RA); organizing pneumonia (OP); Interstitial lung disease (ILD)

A) Check for updates

OPEN ACCESS

\section{Introduction}

In Rheumatoid arthritis (RA), there occurs synovitis of multiple joints that is persistent in nature. Extra-articular involvement is common in this inflammatory joint disease. Extra-articular manifestations in the form of interstitial lung disease (ILD), during the course of RA is seen in about $40 \%$ of patients [1]. The histopathological patterns that occur most frequently in RA include nonspecific interstitial pneumonia (NSIP), usual interstitial pneumonia (UIP), diffuse alveolar damage and organizing pneumonia (OP) [2].

The recent studies recorded the prevalence of interstitial lung diseases in patients of RA as $27 \%$ - 67\%. Although, in these studies it was found that a vast majority of such patients were aymptomatic [3,4]. Organizing pneumonia, in comparison to the other types of ILD in RA is a rare occurrence, with a good prognosis $[5,6]$.

Histologically, OP is characterised by intraluminal buds of granulation tissue present within the alveolar ducts and alveoli [7]. HRCT examinations help in early diagnosis so that timely treatment can be started to reduce the morbidity.

In general, the onset of ILD in RA is preceded by joint manifestations by up to 5 years. We here are presenting a case of 52-year-old male with OP as the first presentation of RA.

\section{Case report}

A 52-year-old male came with history of back pain, nonproductive cough, fever, weight loss. He had no history of any recent travel. There was no history of hypertension, ischaemic heart disease, diabetes mellitus or pulmonary tuberculosis. He was non-smoker and non-alcoholic.

The initial laboratory evaluations were: $\mathrm{Hb} 12.0 \mathrm{~g} / \mathrm{dL}$, TLC 11,900/l, CRP $70.9 \mathrm{mg} / \mathrm{l}$, ESR $58 \mathrm{~mm}$. Renal and liver function tests were within normal limits. Testing for HIV was negative. The chest X-ray revealed left lower zone infiltrate and bilateral areas of consolidation. Mantoux test performed was negative. Sputum for acid fast bacilli (AFB) was negative and on sputum for CBNAAT, Mycobacterium tuberculosis was not detected. Upon persistence of clinical symptoms, HRCT Chest was performed, which showed peribronchial and subpleural bilateral ground-glass opacities with air bronchograms and multiple patchy airspace consolidation. Ziehl-Neelsen (ZN) staining of bronchoalveolar lavage (BAL) tested negative for AFB. Pyogenic and fungal culture was sterile and cytological evaluation revealed lymphocytosis.

Upon further investigation, Rheumatoid factor IgM was 79 $\mathrm{U} / \mathrm{ml}$, IgG $57.6 \mathrm{U} / \mathrm{ml}$, IgA $26 \mathrm{U} / \mathrm{ml}$; antinuclear antibody $<40$ and anticyclic citrullinated peptide antibody (CCP) $43.7 \mathrm{U} / \mathrm{ml}$. 
Transbronchial lung biopsy following pathological examination was made after obtaining patient's consent. Microscopic evaluation of the specimen revealed granulation tissue occupying the alveolar space and terminal bronchioles, with characteristic endoluminal buds of granulation tissue known as Masson bodies, thus establishing the diagnosis of OP (Figures 1,2).

Radiographic examinations of hands, wrist and feet revealed no evidence of arthritis, and there were no articular symptoms at this point in time.

Patient was initiated on methylprednisolone $50 \mathrm{mg} /$ day, following which the patient showed clinico-radiological improvement and was on tapering doses of steroids subsequently. 1 year after the onset, when the patient was on $6 \mathrm{mg}$ of methylprednisolone daily, he developed diffuse arthralgia eventually and was started on Diseasemodifyingantirheumatic drugs therapy (DMARDs) by the rheumatologist, resulting in gradual improvement. Consequently, symptomatically and radiologically patient started improving and is followed-up on regular basis.

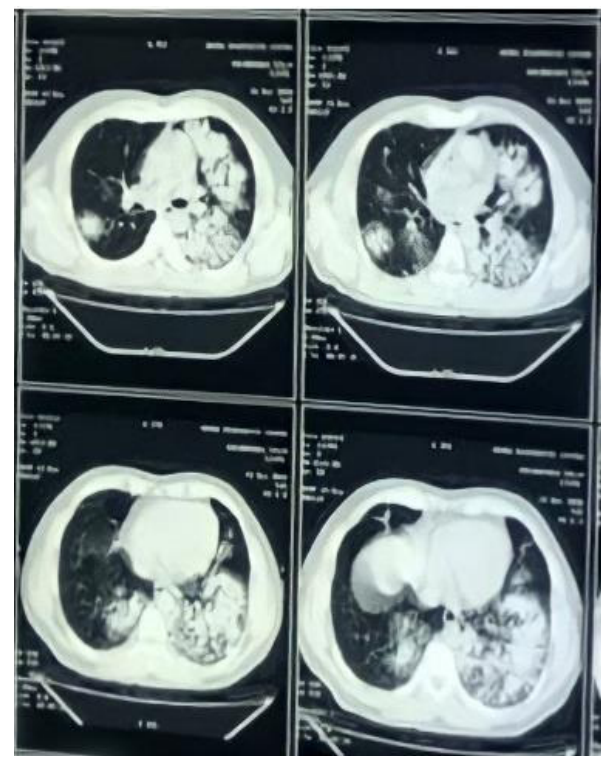

Figure 1: HRCT Chest showing peri-bronchial and subpleural bilateral groundglass opacities with air bronchograms and multiple patchy air space consolidation.

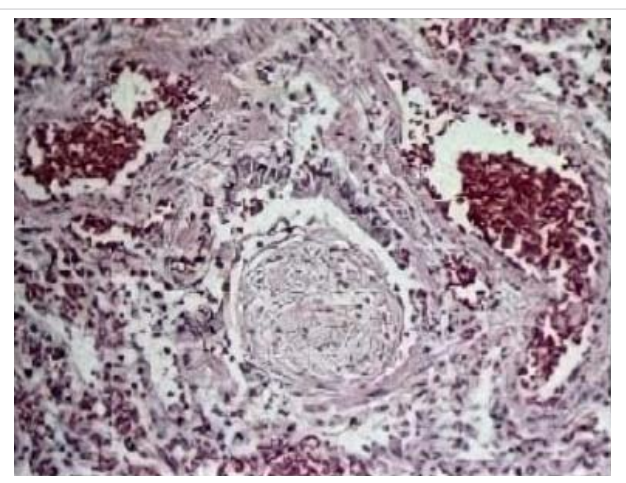

Figure 2: Histopathological evalitions revealed inflammatory debris filling the alveoli and spreading to the alveolar ducts and terminal bronchioles, with characteristic endoluminal buds of granulation tissue known as Masson bodies.

\section{Discussion}

Lung involvement is one of the extra-articular manifestation in RA. The major patterns being non-specific interstitial pneumonia (NSIP), usual interstitial pneumonia (UIP), bronchiolitis and organizing pneumonia (OP). The initial presentation with OP is rare in RA. Although the diagnosis of OP needs histological confirmation, the presence of specific clinical and radiological findings, along with exclusion of other causes can aid the suggestion of OP.

The aetiology of OP can range from pneumococcal pneumonia to several other infectious agents (including bacteria, viruses, fungi and parasites). OP may be seen in association with malignancies like acute myelomonocytic leukaemia, acute lymphoblastic leukaemia, chronic myelomonocytic leukaemia, myelodysplastic syndrome. The iatrogenic organizing pneumonia can be drug or radiationinduced. OP may develop in patients with characterised disorder of unknown cause, such as connective tissue disease. In such diseases, OP may be the main histopathological feature, but a minor proportion may be associated with NSIP pattern.

In all cases where OP is suspected, bronchoalveolar lavage (BAL) should be performed to exclude other diagnoses and to determine the cause of organising pneumonia. An active infection or neoplasms like lymphoma and bronchioloalveolar carcinoma can be disclosed on BAL. On BAL cytological evaluation in OP, there is noted an increase in lymphocytes (20-40\%), neutrophils $(\sim 10 \%)$ and eosinophils $(\sim 5 \%)$, with the lymphocytes higher in level than eosinophils. There usually is a moderate leukocytosis with an increase in neutrophils.

In all patients with OP suspicion, transbronchial lung biopsy should be performed. The histological features comprise of polypoid plugs of loose organizing connective tissue (Masson bodies) within alveolar ducts, alveoli, and bronchioles.

MALT, pulmonary adenocarcinoma, or chronic eosinophilic pneumonia can also produce similar radiological picture [8]. The typical radiological features observed in OP include patchy and multiple areas of airspace consolidation, along with subpleural and peri-bronchial linear and small nodular opacities [9]. The rheumatic organising pneumonia is characterised by "fleeting nature" of lung pneumonic opacities.

It is only in a minor subset of RA patients that pulmonary manifestations precede or occur simultaneous with the joint involvement. In OP cases, including those without joint involvement, serum anti-CCP must be performed to reflect the disease activity. The detection of anti-CCP antibodies in the serum is a significant predictor of RA [10].

The overall prognosis in OP-associated RA is favourable and patients show rapid response to corticosteroids. Effective therapy for OP consists of steroids, with the course varying between 6 to 12 months depending on situations, 
particularly due to frequent relapse after discontinuation of therapy. Patients of OP, who test positive for ACPA should be referred to rheumatologists early for initiation of DMARDs and glucocorticoids to pave their way towards a favourable prognosis.

\section{Conclusion}

Since, OP can be the initial manifestation of RA in rare cases, so patients with OP need to be followed-up cautiously, especially during the initial years if they are either positive for rheumatoid factor or anticyclic citrullinated peptide antibody.

\section{Declaration of patient consent}

Appropriate consent has been obtained from the patient.

\section{References}

1. Cimmino MA, Salvarini $C$, Macchioni $P$, Montecucco $C$, Fossaluzza $V$ et al. Extra-articular manifestations in 587 Italian patients with rheumatoid arthritis. Rheumatol Int. 2000; 19: 213-217.

PubMed: https://pubmed.ncbi.nlm.nih.gov/11063290/

2. Vij R, Noth I, Strek ME. Autoimmune-featured interstitial lung disease: a distinct entity. Chest. 2011; 140: 1292-1299.

PubMed: https://pubmed.ncbi.nlm.nih.gov/21565966/

3. Mori S, Cho I, Koga J, Sugimoto M. Comparison of pulmonary abnormalities on high-resolution computed tomography in patients with early versus longstanding rheumatoid arthritis. J Rheumatol. 2008; 35 : 1513-1521.

PubMed: https://pubmed.ncbi.nlm.nih.gov/18597412/

4. Doyle TJ, Dellaripa PF, Batra K, Frits ML, lannaccone CK, et al. Functional impact of a spectrum of interstial lung abnormalities in rheumatoid arthritis. Chest. 2014; 146: 41-50. PubMed: https://pubmed.ncbi.nlm.nih.gov/24305643/

5. Zou YQ, Li YS, Ding XN, Ying ZH. The clinical significance of HRCT in evaluation of patients with rheumatoid arthritis-associated interstitial lung disease: a report from China. Rheumatol Int. 2012; 32: 669-673. PubMed: https://pubmed.ncbi.nlm.nih.gov/21132550/

6. Kim EJ, Collard HR, King TE. Rheumatoid arthritis-associated ILD: the relevance of histopathological and radiographic pattern. Chest. 2009; 136: 1397-1405.

PubMed: https://pubmed.ncbi.nlm.nih.gov/19892679/

7. Colby TV. Pathological aspects of bronchiolitis obliterans organizing pneumonia. Chest. 1992; 102: 38-43. PubMed: https://pubmed.ncbi.nlm.nih.gov/1623809/

8. Baque-Juston M, Pellegrin A, Leroy $S$, Marquette $\mathrm{CH}$, Padovani B. Organizing pneumonia: What is it? A conceptual approach and pictorial review. Diagn Interv Imaging. 2014; 95: 771-777. PubMed: https://pubmed.ncbi.nlm.nih.gov/24559802/

9. Izumi T, Kitaichi M, Nishimura K, Nagai S. Bronchiolitis obliterans organizing pneumonia. Clinical features and differential diagnosis. Chest. 1992; 102: 715-719.

PubMed: https://pubmed.ncbi.nlm.nih.gov/1516392/

10. Lee DM, Schur Ph. Clinical utility of the anti-CCP assay in patients with rheumatic diseases. Ann Rheum Dis. 2003; 62: 870-874. PubMed: https://pubmed.ncbi.nlm.nih.gov/12922961/ 